\title{
Evaluation of Rosenbaum's Proposal for Locally Increasing the Effective Vertical Emittance of the APS Beam
}

\author{
M. Borland, L. Emery, N. Sereno \\ $\mathrm{AOD} / \mathrm{OAG}$ \\ June 2002
}

\section{Introduction}

At the APS Retreat in May 2002, G. Rosenbaum proposed a method for increasing the effective vertical emittance of the APS beam. His proposal, illustrated in Figure 1, is to use a rapidly-modulated four-magnet vertical position bump in a single straight section. The proposed modulation frequency is a $10-\mathrm{kHz}$ triangle wave, with a kick angle of 0.65 mrad. There are several aspects of this proposal that may be problematical:

1. The high modulation frequency will require the use of a ceramic chamber with a thin conductive coating, similar to what is used for the injection kicker magnets. This is not necessarily a problem, but the following aspects need to be examined:

(a)The chamber coating needs to be specified correctly to ensure that the beam motion reflects the desired triangle-wave pattern.

(b)The effect of the eddy currents in the chamber on the multipole content of the magnetic field needs to be computed, to ensure that the system does not have undesirable effects on the beam.

2. The magnets and their chambers will require careful matching in order to ensure that the beam is not disturbed outside of the bump. We know from experience with the APS kickers that this can be difficult.

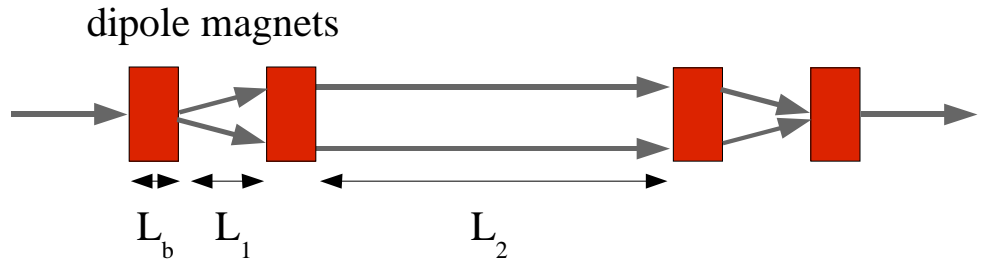

Figure 1: Illustration of Rosenbaum's scheme. Arrows show the extremal paths of the electron beam in the vertical plane.

These issues are examined in the remainder of this note. The magnet and power supply designs are beyond our scope. We simply point out that these will not be conventional magnets or power supplies. The magnets will almost certainly need to be of ferrite construction, similar to the injection kickers. The power supplies will be unique and unlike anything else in the APS ring. 


\section{Chamber Requirements to Preserve Desired Waveform}

The desired beam motion is a triangle wave at $10 \mathrm{kHz}$. This is preferred over a sinusoidal modulation as the latter produces a dumbell-shaped beam distribution. A triangle-wave pattern will produce an effective beam distribution that looks like a flat-top with Gaussian ends. We start with the series expansion for a triangle wave:

$$
B_{e}(t)=8 \frac{B_{p e a k}}{\pi^{2}} \sum_{m=0}^{\infty} \frac{(-1)^{m}}{(2 * m+1)^{2}} \sin \left(\omega_{o}(2 * m+1) t\right)=\sum_{m=0}^{\infty} B_{m} \sin \left(\omega_{m} t\right),
$$

where $\omega_{\mathrm{o}}$ is the fundamental angular frequency of the waveform, $B_{e}(t)$ is the external magnetic field at time $t$, and $B_{\text {peak }}$ is the peak value of the imposed magnetic field. The attenuation and phase shift of the magnetic field due to the chamber coating is different for each harmonic.

Results for a single frequency are given by S. Kim [1]. In particular, for an external magnetic field $B_{m} \sin \left(\omega_{m} t\right)$, the steady-state field inside the chamber is

$$
B_{m, i}(t)=B_{m} \frac{1}{\sqrt{\left(1+\left(\omega_{m} \tau\right)^{2}\right)}} \sin \left(\omega_{m} t-\phi_{m}\right),
$$

where $\tau$ is the exponential time constant for field penetration and $\phi_{m}=\arctan \left(\omega_{m} \tau\right)$ is the phase shift for the $m^{\text {th }}$ harmonic. To find the total field $B_{i}(t)$ inside the chamber, we simply sum the $B_{m, i}(t)$. The time constant $\tau$ is given by

$$
\tau=\frac{\mu_{o} h_{y}}{2 R_{s}} F\left(h_{x}, h_{y}\right),
$$

where $h_{x}\left(h_{y}\right)$ is the half-width of the chamber in the horizontal (vertical). The field, of course, is horizontal. $F\left(h_{x}, h_{y}\right)$ is a complicated function with a range of 1 to 1.5 for elliptical chambers. To be conservative (we believe), we will assume $F=1.5$. Note that $F=1$ for a round chamber.

An idea worth exploring is to make the chamber rectangular (with the same internal aperture as the ID chamber) with top and bottom walls made of metal, and the side walls ceramic coated with metallic paint. The horizontal walls, having high conductivity, will contribute less resistive wall impedance than a coated ceramic. Also, because the field is parallel to the metal walls, there will be no eddy currents produced on them. We don't think such a chamber has been made before. Therefore we don't know whether the metalto-ceramic bond along the length of the chamber can be made strong enough to hold vacuum under normal handling. There will be some induced currents on the coated ceramic sides. S. Kim [2] gives the same expression for the time constant as in an elliptical tune except that the $F$ would be of the order $\left(h_{y} / h_{x}\right)^{3}$, which is pretty small. It seems that there is a great advantage in using a rectangular chamber over an elliptical one. What is not included in the theory is the interaction between the eddy currents in the coating and the metal sides. A full, finite-element calculation is necessary for this rectangular aperture geometry, with a determination of angular tilt alignment tolerance. For the rest of the note, an elliptical chamber will be assumed. 
$R_{s}$ is the sheet resistance, given by $R_{s}=\rho_{r} / d$, where $\rho_{r}$ is the resistivity and $d$ is the thickness of the resistive coating. For the APS kicker chambers [3], $R_{s}$ was chosen to be $0.1 \mathrm{Ohm}$, with a tolerance of $20 \%$. Ideally, the tolerance should be $2 \%$, but this is not achievable with present coating technology. A $10 \%$ tolerance was specified for replacement chambers to improve the kicker match. The value of $R_{s}$ must also be evaluated to ensure that resistive heating due to the beam current is not excessive. Because the waveform for Rosenbaum's proposal is much slower than the APS kicker pulse, $R_{s}$ can be smaller, which means the resistive losses will be smaller. Hence, we do not need to worry about this issue.

We wrote a script that allows computation of $B_{i}(t)$ for various values of $R_{s}$. It is desirable to minimize the number of vacuum chamber transitions. Hence, the ceramic chambers will ideally have the same vertical and horizontal aperture as the ID chamber. We assume $h_{y}=4 \mathrm{~mm}$. The transition from a rectangular chamber to an elliptical ID chamber will not produce a significant impedance. Our (somewhat arbitrary) criterion for the magnetic field inside the chamber is that the peak internal magnetic field should not deviate by more than $1 \%$ from the peak external magnetic field.

Figure 2 shows a series of waveforms for the magnetic field inside the chamber, normalized to the peak magnetic field outside the chamber. One sees that as $R_{s}$ becomes larger, the internal field resembles a triangle wave more closely. It is useful to note that $R_{\mathrm{S}}=7.2 \times 10^{-4} \mathrm{Ohm}$ for a stainless steel chamber of $1-\mathrm{mm}$ thickness. Hence, it is impractical to imagine using a totally metal chamber. Figure 3 shows the fractional deviation of the peak internal field from the peak external field as a function of $R_{s}$. With the reservation that there is some uncertainty introduced by lack of precise knowledge of $F\left(h_{x}, h_{y}\right)$, we conclude that $R_{s} \geq 0.01 \mathrm{Ohm}$ is acceptable. To prevent excessive heating of the chamber by the beam current, $R_{s} \geq 0.1 \mathrm{Ohm}$ is recommended, as for the present kicker chambers. The corresponding time constant will be $38 \mathrm{~ns}$, which is very short compared to the waveform period. 


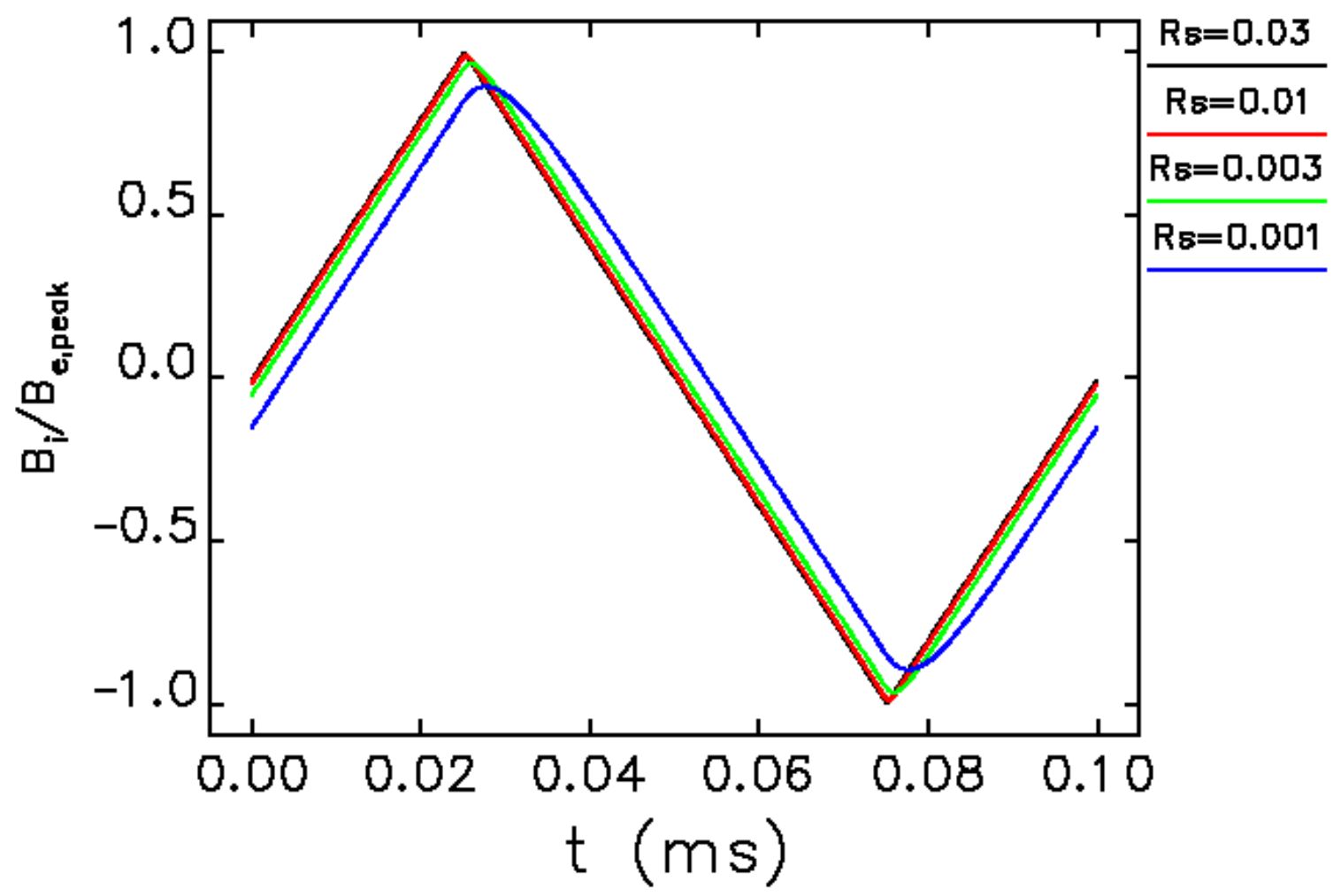

Figure 2: Internal magnetic field as a function of time for various values of $R_{s .}$ The waveforms are computed by summing the series for 200 harmonics, assuming $F=1.5$. 


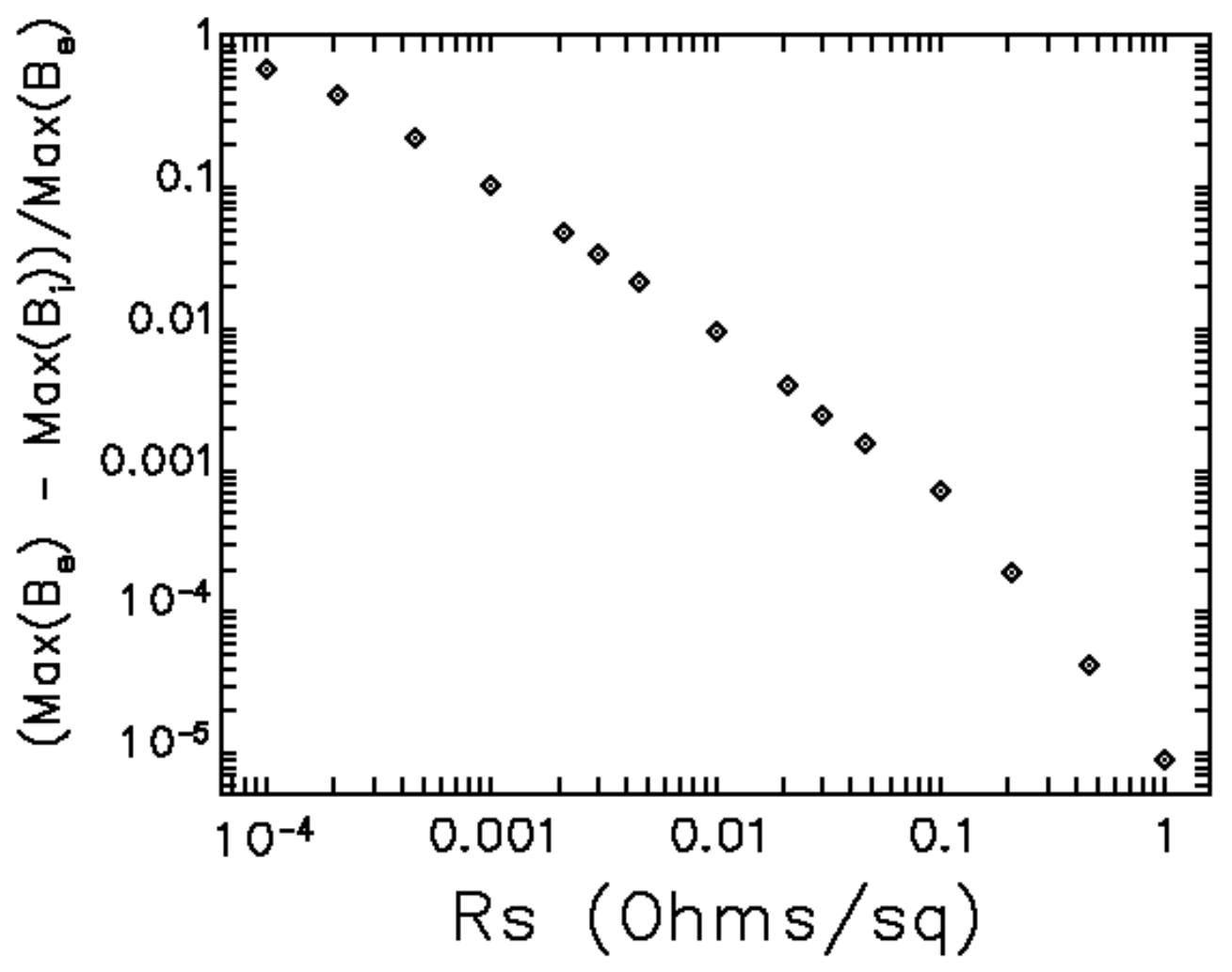

Figure 3: Fractional deviation of maximum internal magnetic field from maximum external magnetic field as a function of $R_{s,}$ assuming $F=1.5$.

\section{Multipole Content of the Internal Magnetic Field}

In addition to the shielding of the dipole field considered above, there is an eddy-currentinduced sextupole field for an elliptical chamber. The sextupole multipole strength can be expressed in terms of an integral over the chamber cross section. For this calculation we use a thickness $d=0.004 \mathrm{~mm}$ that corresponds to $R_{s}=0.1 \mathrm{Ohm}$, and the geometry for the 8-mm chamber, which has a major ellipse axis of $40 \mathrm{~mm}$ and minor axis length of 8 $\mathrm{mm}$. For $7 \mathrm{GeV}$, the sextupole effective $K_{2} L$ value assuming $L=0.1 \mathrm{~m}$ is $0.005 \mathrm{~m}^{-1}$. This is negligibly small compared to the APS storage ring sextupole integrated strength of 3 $\mathrm{m}^{-1}$ at $7 \mathrm{GeV}$. The eddy-current-induced sextupole fields for all 35 sectors filled with raster dipoles is therefore negligibly small compared to the field for the storage ring sextupoles.

A similar calculation of the eddy-current distribution induced in the elliptical chamber cross section can be used to estimate the power deposited per unit chamber length (along the beam direction). The power density is given by the eddy-current density times the induced electric field. The integral of the power density over the chamber cross section 
gives $74.2 \mathrm{~W} / \mathrm{m}$ for the elliptical geometry used in the induced sextupole calculation and $R_{s}=0.1 \mathrm{Ohm}$. This will result in a power of approximately 15 watts for a $20-\mathrm{cm}-$ long chamber, which is not an excessive heat load.

\section{Magnets}

Here are some comments on the magnets. The required amplitude of the oscillation is $0.25 \mathrm{~mm}$. For a 5-m-long straight section with one 2.4-m undulator, there is room for two 1.3-m-long spaces upstream and downstream of the undulator. Spacing the corrector centers by $1.2 \mathrm{~m}$, instead of the $0.3 \mathrm{~m}$ proposed originally, will decrease the required angle by a factor of four, which is of great benefit. The angle is $(0.25 \mathrm{~mm}) /(1.2 \mathrm{~m})=0.2$ mrad. For a $0.1-\mathrm{m}-$ long magnet, the magnetic field is $0.047 \mathrm{~T}$ or $470 \mathrm{G}$. For a horizontal gap of $45 \mathrm{~mm}$, say, the drive current will be 1700 A-turns.

\section{Tolerances on Chambers and Power Supplies}

There are no tolerances given in Rosenbaum's proposal for the sharpness of the triangular wave. We can make an estimate of what photon intensity nonuniformity would result from a flattening of a certain short duration at the peaks of the triangular wave. The vertical source size for a $1 \%$ emittance ratio beam is $13 \mu \mathrm{m}$. The range in vertical position is $\pm 250 \mu \mathrm{m}$. Therefore, the vertical beam spot is spread out 40 -fold. If the beam should linger at a peak for about $1 / 40$ of a half-period (i.e., $1.2 \mu \mathrm{s}$, less than a revolution period) before turning back, then the intensity at the peak of the motion would be twice that of the average. However, the time constant for the proposed chamber is $38 \mathrm{~ns}$, much smaller than the $1.2 \mu$ above. Therefore we don't expect much intensity modulation.

In passing, it is worth noting that because of the high frequency of the oscillation, the bunch pattern is potentially relevant. For the singlets fill pattern, the bunch spacing is $150 \mathrm{~ns}$, or 1/300 of the half-period of the oscillation. Hence, the vertical position of successive bunches will change by $1.5 \mu \mathrm{m}$, which is about $10 \%$ of the vertical beam size. For the hybrid pattern, however, the bunch spacing is not uniform as the beam is concentrated on two sides of the ring. The change in vertical position for the bulk of the beam (excluding the singlet) from turn to turn will be about $37 \mu \mathrm{m}$, or about three times the beam size. Therefore the beam will appear banded in the vertical plane, which may produce undesirable characteristics if the beam is imaged. One way to mitigate such problems is to reduce the frequency or reduce the size of the oscillation, or some combination thereof.

If the four magnets and their power supplies couldn't produce a mathematically exact triangular wave, then, for the sake of preserving the emittance for other users, the four correctors should produce the same waveform within some tolerance. A general mismatch of three correctors relative to a reference corrector would create a trajectory perturbation characterized by two parameters, a position and angle error at the end of the 
straight section, producing a global effective emittance increase. The third parameter would be an angle error on the trajectory inside the undulator, which is a minor concern compared to the global emittance increase.

The mismatch can be due to power supply mismatch (if we use individual power supplies for each corrector), vacuum chamber mismatch, and variations in the magnet construction.

If we have $R_{s}=0.1 \mathrm{Ohm} / \mathrm{sq}$, then the fractional shielding of the field is about 0.001 (see Figure 3), much less than $1 \%$. Errors in $R_{s}$ must be very large to reach a fractional shielding of 0.01 . Therefore there is no problem for the vacuum chamber to meet the specification of $1 \%$. (The same conclusion is reached when we realize that the time constant is as small as $38 \mathrm{~ns}$. A variation in time constant at that magnitude isn't expected to shift the phase significantly.)

If one of the correctors is not matched with the others by $1 \%$ of the peak at any time during the triangular waveform, then the vertical angle error will be $2.0 \mu \mathrm{ad}$. As the beam circulates over many turns, the beam at other ID light source points will appear to shake with about a 5- $\mu \mathrm{m}$ amplitude, or $2.5 \mu \mathrm{m}$ rms. This is to be compared with the vertical source size of $13 \mu \mathrm{m}$ for the standard low-emittance lattice running at $1 \%$ coupling. Since the users have accepted an emittance ratio of $2 \%$ in the last two runs, and we used to operate with a $1 \%$ emittance ratio, it seems that some increase in vertical source size is acceptable. For the purpose of illustration, let's make the tolerance equal to one half the vertical source size mentioned above, that is, a $7-\mu \mathrm{m}$ rms vertical motion observed at the ID source points.

With this tolerance of $7 \mu \mathrm{m}$ rms, we get a magnet mismatch of tolerance $2.8 \%$ of the peak. Note that this mismatch is for any time duration during the cycle, i.e., once a mismatch occurs in the course of a $10-\mathrm{kHz}$ cycle, the oscillation will persist because the cycle repeats and is shorter than the damping time. Dividing this tolerance equally between the power supply, vacuum chamber, and magnet construction (an arbitrary division), we get about $1 \%$ tolerance on each.

We have a choice between specifying a strict tolerance for the matching of the fields of the magnets with no plans for waveform correction, and specifying looser tolerances for the magnetic fields with the further possibility of correcting the errors.

\section{Correction of Waveforms}

A small corrector mismatch could be measured using storage ring BPM turn-by-turn data. However, because the damping time of the centroid motion is $10 \mathrm{~ms}$, or 100 times the excitation period, a BPM signal would contain the history of the previous 100 driving oscillations. Thus it may be difficult to extract the errors in the waveforms from the BPM signal. A sophisticated inversion algorithm would have to be developed that includes the removal of the turn-by-turn readback noise of the BPMs, which is about $100 \mu \mathrm{m}$, a large 
number compared to the expected mismatch oscillations. Another complication is the is the time constant of the response of the power supply/corrector/vacuum chamber system into the inversion.

We've had a somewhat difficult experience correcting the perturbations of circularly polarizing undulator (CPU) in ID4. Here the perturbation time scale is longer by about a thousand, so there is no problem connected with the damping time as above, and the inversion of BPM signal to get the required correction is simpler. The waveform corrections to be applied are smooth functions of about $50 \mathrm{~ms}$ duration with structures of about $5 \mathrm{~ms}$. The corrector response time, which is also a major complication here, is about $7 \mathrm{~ms}$. Unfortunately, the errors couldn't be corrected accurately by the inversion algorithm. The problem in convergence may have been due to the relative inaccuracy of the calibration of the corrector to BPM response, which manifests itself when correcting a second integral error with two independent correctors. (We mention this here since this problem may occur in correcting the $10-\mathrm{kHz}$ second integral error.) The CPU

perturbations have finally been corrected by about a factor of 20 after about 100 iterations of manipulating waveforms points manually using a subjective penalty function. We believe the same results would have been achieved for the CPU with the inversion algorithm if we had corrected the first integral error only. We expect a more complicated experience overall in the matching of the $10-\mathrm{kHz}$ magnets, not to mention more machine physics studies time.

The real-time feedback correction system, with a sampling time of $600 \mu \mathrm{s}$, will not be useful in this project.

We advise that we not rely on correction, but fabricate the magnets and power supplies with the tighter tolerances. In that case we no longer require independent supplies. If it is easier to power all magnets in series, then we should do so.

\section{Conclusion}

We think that this scheme has no serious flaws in the beam physics aspects. We suggest that as much of the straight section as possible should be used to create the vertical bump. Four ceramic chambers would need to be installed in the straight section. The tolerance for the magnet and the power supplies is about $1 \%$ each. This is based on allowing an increase in the emittance ratio of 0.005 . The tolerance for the resistivity of the ceramic chamber coating is easy to achieve. The eddy currents in the ceramic chambers produce very little sextupole component. If we use only one power supply to power all magnets in series, then only the magnets and cabling require a tolerance in matching strength. The tolerance would then be about $2 \%$.

We don't think relying on waveform correction is a good idea, based on past experiences. More work needs to be done on the feasibility of the design of the magnets, vacuum 
chamber, and the power supplies.

Finally, if banding problems in certain fill patterns are an issue for the users of the beamline, then we suggest lowering the oscillation frequency by a factor of 10 , to $1 \mathrm{kHz}$. A system with variable oscillation frequency might be ideal, but this would complicate matching of cables, magnets, and power supplies.

\section{References}

[1] S. Kim, "Calculation of Pulsed Kicker Magnetic Field Attenuation Inside Beam Chambers," APS Light Source Note LS-291, January 8, 2001

[2] S. Kim, private communication.

[3] C. Doose et al., "Investigation of the Surface Resistivity Tolerance of the Kicker

Ceramic Vacuum Chamber at APS," Proceedings of the 2001 PAC, 1491 (2001). 